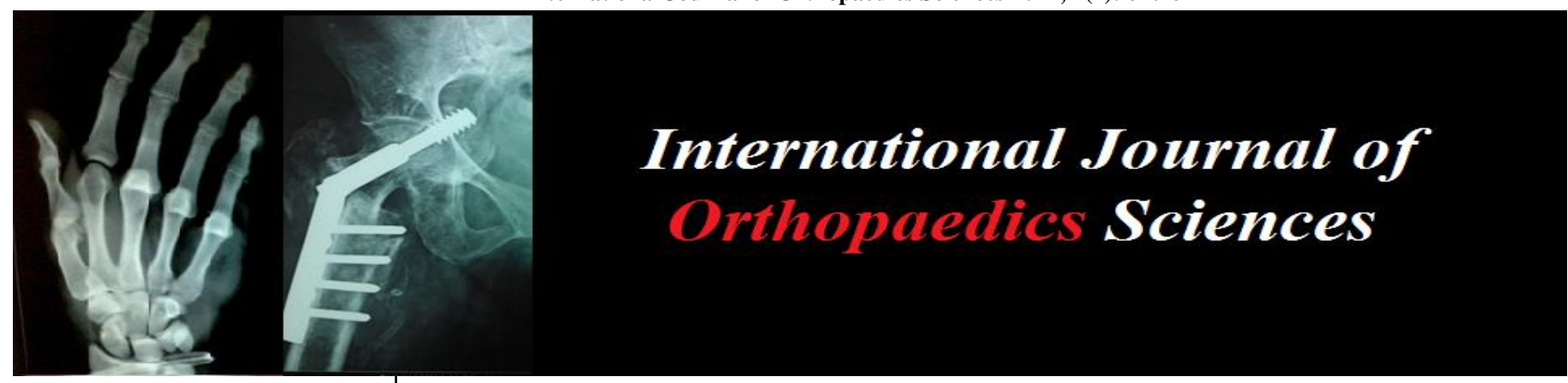

E-ISSN: 2395-1958

P-ISSN: 2706-6630

IJOS 2021; 7(1): 620-624

(C) 2021 IJOS

www.orthopaper.com

Received: 25-11-2020

Accepted: 27-12-2020

Dr. Kaushik YS

St. John's Medical College,

Bengaluru, Karnataka, India

Dr. Sandesh GM

St. John's Medical College,

Bengaluru, Karnataka, India

Dr. Joseph Eugene Nidhiry

St. John's Medical College,

Bengaluru, Karnataka, India

Dr. Rinju Krishnan

St. John's Medical College,

Bengaluru, Karnataka, India

Dr. Tarun Desai

St. John's Medical College,

Bengaluru, Karnataka, India

Dr. Surendra Babu

St. John's Medical College,

Bengaluru, Karnataka, India

Corresponding Author:

Dr. Sandesh GM

St. John's Medical College,

Bengaluru, Karnataka, India

\section{Twin interlocking cephalomedullary nail vs PFN for unstable intertrochanteric fractures: Short term outcome, an Indian perspective}

\author{
Dr. Kaushik YS, Dr. Sandesh GM, Dr. Joseph Eugene Nidhiry, Dr. Rinju \\ Krishnan, Dr. Tarun Desai and Dr. Surendra Babu
}

DOI: https://doi.org/10.22271/ortho.2021.v7.i1j.2551

\section{Abstract}

Objective: The main objective of the study was to compare proximal femoral nail and Twin interlocking cephalomedullary nail in terms of short-term functional and radiological outcome.

Methods: 30 patients with unstable intertrochanteric femur fractures operated by a single surgeon were included in the study. 15 in the PFN group and 15 in the Twin interlocking cephalomedullary nail group. Modified Harris Hip score was used to assess the functional outcome.

Results: The time to union and the functional outcome was comparable in both the groups and desired linear compression of up to $10 \mathrm{~mm}$ at the fracture site could be achieved in the twin interlocking cephalomedullary nail group.

Conclusion: Twin interlocking Cephalomedullary nail is a very good alternative to PFN in treatment of Unstable Intertrochanteric fractures of the Femur.

Keywords: Unstable, intertrochanteric, PFN, twin interlocking cephalomedullary nail

\section{Introduction}

Unstable Intertrochanteric fractures are common fractures and they are challenging to manage due to the difficulties faced to achieve anatomic reduction and stable fixation ${ }^{[1-3]}$.

In the present day, intertrochanteric fractures are treated by operative management using both extramedullary and intramedullary implants. The advantage of operative management is early rehabilitation, providing a stable, mobile and a painless hip ${ }^{[4-5]}$.

For stable fractures extramedullary devices like Dynamic Hip screw have been used successfully with excellent clinical and functional outcomes. But in management of unstable intertrochanteric fractures use of intramedullary devices have shown to have superior clinical and functional outcomes as they are biomechanically better suited for this fracture type ${ }^{[6-8]}$.

Many intramedullary deigns have been used to treat unstable intertrochanteric fractures. But there is paucity of literature regarding which implant is best in management of these fractures. The purpose of this study was to compare Proximal femoral nail (AO) which has been used extensively and a Twin interlocking cephalomedullary nail (InterTAN by Smith and Nephew, HFN by Syncera) a relatively new implant, regarding short term functional outcomes in adults with Unstable intertrochanteric fractures.

The Twin interlocking cephalomedullary nail has a trapezoidal proximal shape, and uses two integrated lag screws with an effective size of $15.25 \mathrm{~mm}$ for femoral head fixation. The proximal nail shape and 'the rack and pinion design' of the nested screws give excellent rotational stability and the ability for controlled fracture compression ${ }^{[9]}$.

\section{Materials and Methods}

It was a prospective study.

30 patients who had unstable intertrochanteric fractures (AO type A2 and A3) which were comparable with respect to baseline characteristics were chosen between JAN 2018 - JUNE 2020; The patients were divided into two groups based on the implant used for the surgery. One group of 15 patients with Proximal Femur Nail and 15 patients with Twin interlocking 
cephalomedullary nail. The choice of implant to be used was randomly selected by the operating surgeon. All patients selected in this study were operated by a single orthopaedic surgeon.

\section{Inclusion criteria}

1. Patients with unstable intertrochanteric fractures $(\mathrm{AO}$ type A2 and A3).

\section{Exclusion criteria}

1. Patients with pathological fractures.

2. Patients operated with implants other than PFN and Twin interlocking cephalomedullary nail.

3. Revision surgery.

4. Any active infection around the hip joint.

All fractures were classified according to A O classification. Surgical technique,

All patients were positioned in supine position on a fracture table, closed manipulation done under fluoroscopic guidance, parts were painted and draped under strict aseptic precautions. Approximately 2 -inch length incision was made proximal to the tip of trochanter. The guide-wire was inserted into the medullary canal from the tip of trochanter and its position was controlled in both anterior and lateral views. Serial reaming of the proximal femur was done. The guide wire was removed after the intramedullary nail inserted over the wire. After the confirmation of anteversion of the nail, two proximal locking screws in proper length were inserted. Distal locking was done using the jig.

\section{For PFN}

Two different screws, one $8 \mathrm{~mm}$ lag screw was inserted inferiorly and superiorly a $6.5 \mathrm{~mm}$ anti rotation screw was inserted which was $10 \mathrm{~mm}$ shorter than the lag screw.

\section{For twin interlocking intramedullary nail}

Two integrated proximal screws, which are the lag screw in a diameter of $11 \mathrm{~mm}$ and the compression screw in a diameter of $7 \mathrm{~mm}$ (as a rule $5 \mathrm{~mm}$ shorter then lag screw length). Firstly, the Lag screw was inserted with the antirotation bar held in place and then the compression screw is inserted and desirable linear compression at the fracture site is achieved up to $10 \mathrm{~mm}$. Additionally SET screw could be placed once good reduction was achieved and compression was attained at fracture site using cephalomedullary screws to prevent uncontrolled sliding at the fracture site post operatively.

Immediate postoperative $\mathrm{x}$-rays were taken and quality of reduction was assessed by Baumgaertner's criteria (fig 1) ${ }^{[10]}$. Standard rehabilitation protocol was followed for both groups. Antibiotics and DVT prophylaxis were given to all patients. All patients were followed up at 1 month, 3 month and at 6 months postoperatively. Functional outcome was Assessed by using modified Harris Hip Score and. X-rays were taken to asses union at every follow up.

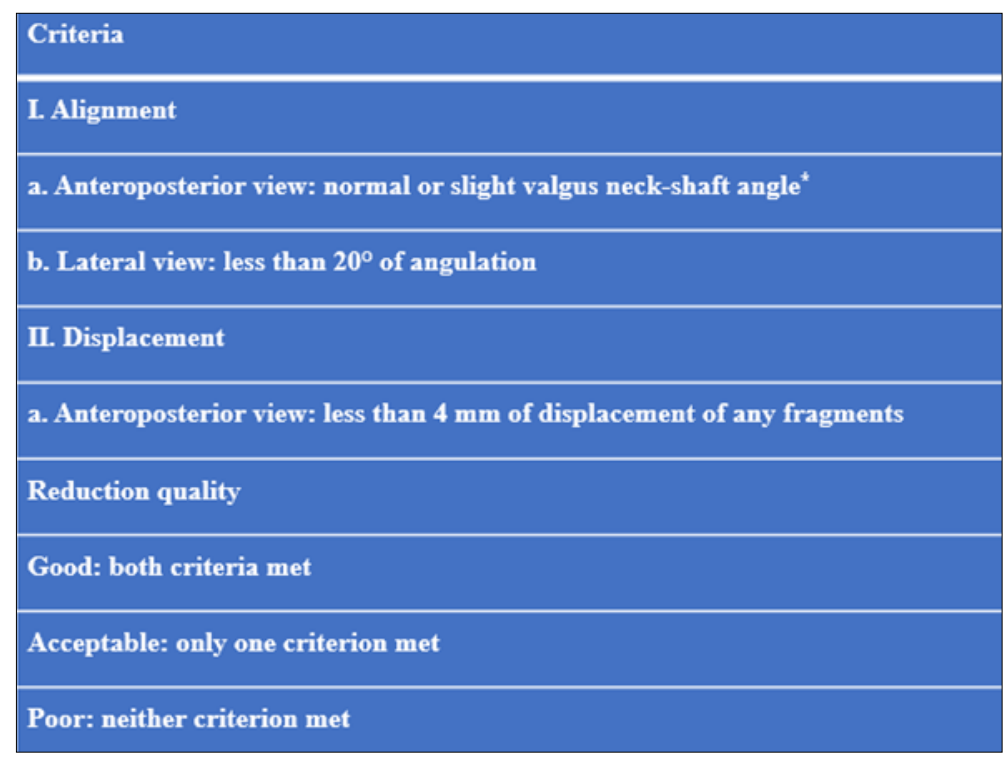

Fig 1: Baumgeartner's reduction quality criteria

\section{Results}

A total of 30 patients were included in the study. 15 patients in the PFN group and 15 patients in the Twin interlocking cephalomedullary nail group. Table one shows the comparison of baseline characteristics between two groups.

Table 1: Baseline characteristics

\begin{tabular}{|c|c|c|}
\hline & PFN & InterTAN \\
\hline Age (Years) & 64 & 67.5 \\
\hline Sex - Male & 09 & 06 \\
Female & 10 & 05 \\
\hline Side of injury- Left & 07 & 04 \\
Right & 08 & 11 \\
\hline Mode of injury- Slip and fall RTA & 12 & 13 \\
\hline Fracture type (AO/OTA) & 03 & 02 \\
A2 & 10 & 8 \\
A3 & 05 & 7 \\
\hline
\end{tabular}


Table 2: Intra and post-operative variables

\begin{tabular}{|c|c|c|}
\hline & PFN & InterTAN \\
\hline Duration of surgery (Avg. in min) & 52 & Post op day 3 \\
\hline Time to mobilization (Days) & Postop day 3 & 6 \\
\hline Average Time to full weight bearing (Avg. in weeks) & 8 & 17 \\
\hline Average Time to union (Weeks) & 18.5 & 13 \\
\hline Baumgaertner criteria for reduction & 12 & 02 \\
Good & 03 & 00 \\
Acceptable & 00 & \\
Poor & & \\
\hline
\end{tabular}
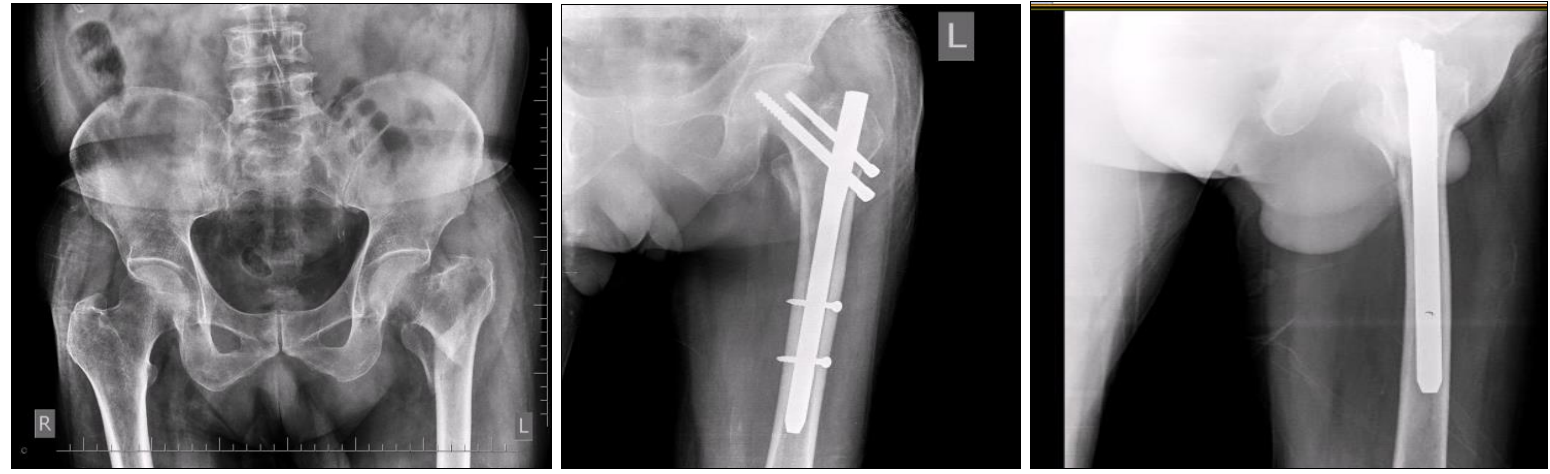

Fig 2: Proximal femoral nail
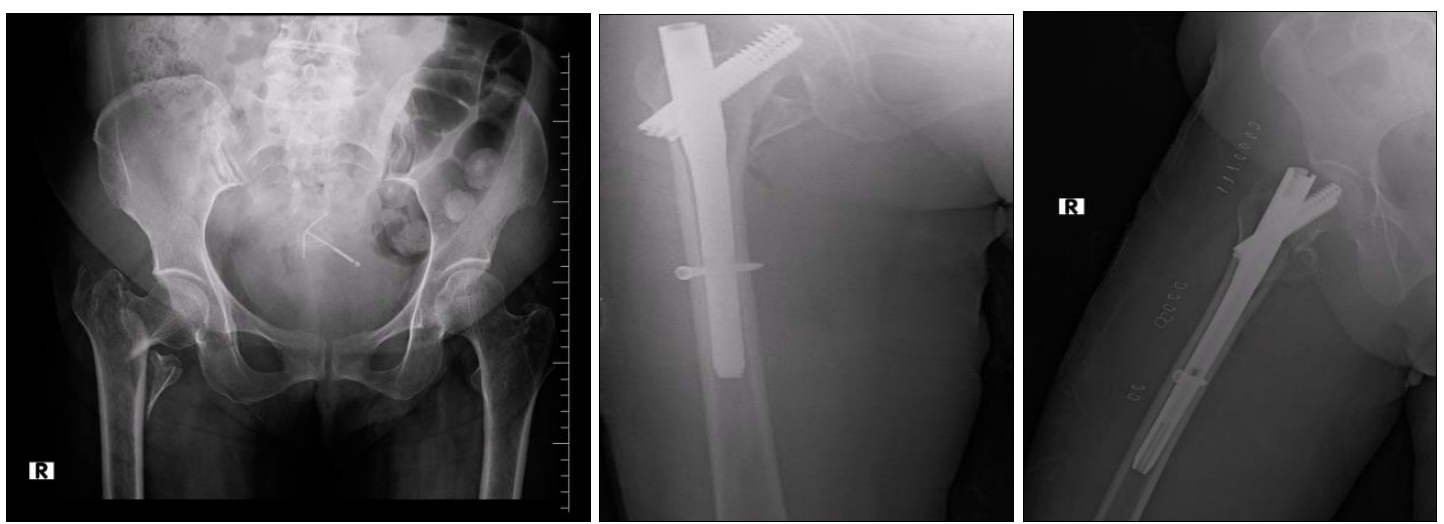

Fig 3: Twin interlocking cephalomedullary nail

Time to union between the two groups i.e., PFN (18.5 weeks) and Twin interlocking cephalomedullary nail (17 weeks) was statistically insignificant.

Functional outcome at the end of 6 months was assessed by Modified Harris Hip score it was good to excellent in 13/15 cases, fair in $2 / 15$ cases in the twin interlocking nail group and good to excellent in $12 / 15$ cases, fair in $3 / 15$ cases. The difference between the two groups was statistically insignificant.

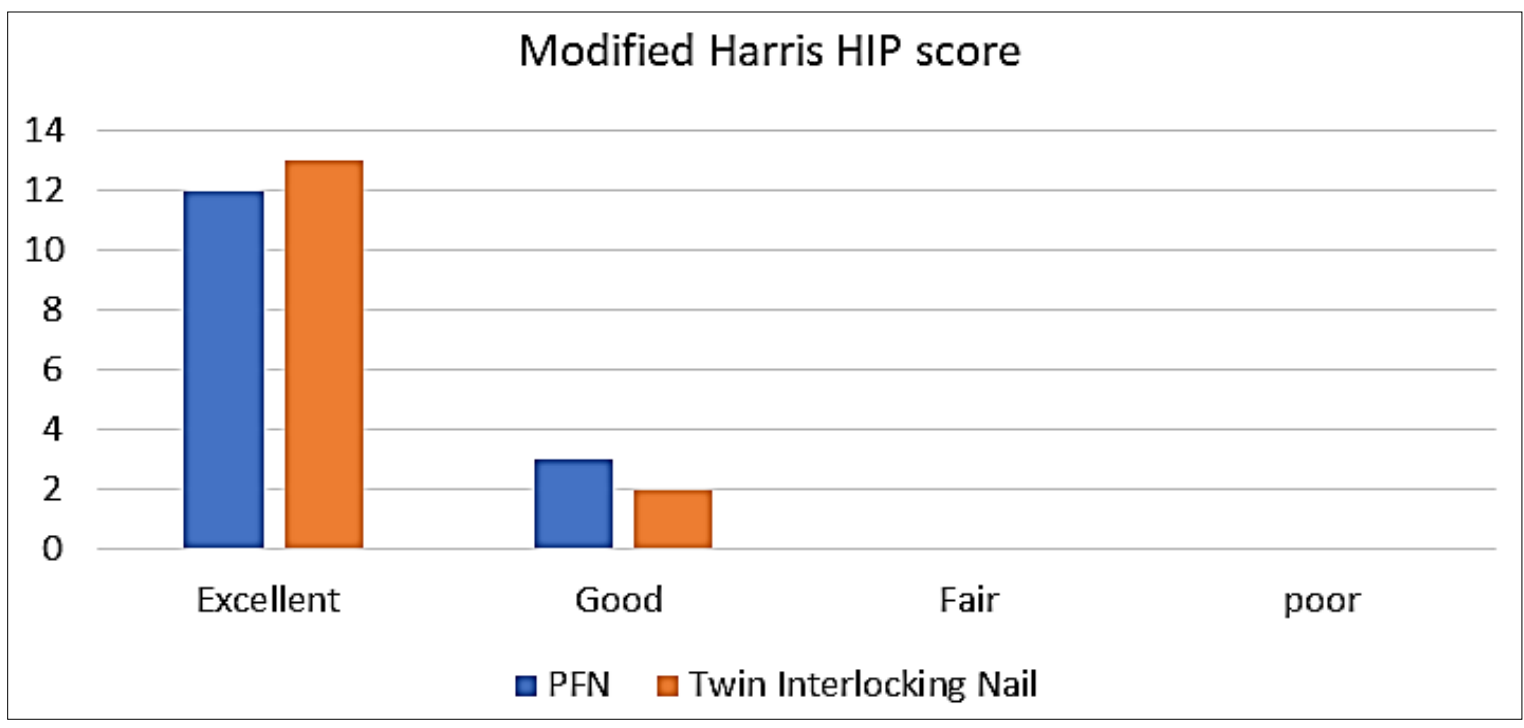

Fig 4: Modified harris hip score 
Coming to the complications, we observed rotation of the cervical fragment intraoperatively in 1 case of PFN group when the proximal guide wire was not placed correctly.

There was superficial wound infection noticed in 1 case of PFN group and 1 case of Twin interlocking cephalomedullary nail which resolved with dressing and a course of oral antibiotics.

There was 1 patient with $\mathrm{Z}$ effect in PFN group and revision surgery was done for the same and patient is doing fine.

There was 1 patient with varus malunion in the PFN group and the patient was functionally doing good so nothing was done for the same.

\section{Discussion}

The biomechanical superiority of intramedullary devices has long been established in treatment of unstable intertrochanteric fractures ${ }^{[11]}$. In our study we tried to explore the Indian perspective in the management of unstable intertrochanteric fractures by comparing two different intramedullary nails in view of short-term functional outcome. We compared PFN and a relatively new cephalomedullary nail integrated twin interlocking screws.

PFN, Proximal Femoral Nail, developed by AO/ASIF, a thirdgeneration load sharing device works on the principle of controlled fracture collapse and is closer to the mechanical axis of the femur and a shorter lever arm, has been used extensively in the treatment of these fractures and has advantages of less soft tissue dissection and addition of 6.5 $\mathrm{mm}$ anti-rotation screw to prevent the rotation of the proximal fragment ${ }^{[12-15]}$.

But well-known complications of this device are implant related problems like varus collapse, screw cut out, $\mathrm{Z}$ effect, Reverse $\mathrm{Z}$ effect, and inability to put an anti-rotation screw in some cases due to a narrow neck has been documented ${ }^{[12]}$.

Keeping this in mind, the twin integrated interlocking cephalomedullary nail was introduced, which has a has a trapezoidal proximal shape, and uses two integrated lag screws with an effective size of $15.25 \mathrm{~mm}$ for femoral head fixation. The proximal nail shape and the rack and pinion design' of the nested screws give excellent rotational stability and the ability for controlled fracture compression ${ }^{[16]}$

In our study fracture healing was assessed clinically and radiologically. The average time for union in the PFN group was 18.5 weeks and, in the twin, interlocking integrated cephalomedullary group was 17 weeks. This was statistically insignificant but the early union in case of the latter group may be attributed to the nail design as explained above. Similar times to union were observed in the other studies. Min et al. ${ }^{[17]}$. studied reverse oblique fracture patterns and treated them with PFN or gamma nail and observed bone union time as averaging between 16.5 weeks in PFN group and 17.9 weeks in gamma nail group.

All patients were followed up for a minimum of 6 months in our study and no patients were lost to follow up. Functional outcome at final follow up was assessed by modified Harris hip score and we found good to excellent results in 13/ 15 patients in Twin integrated interlocking cephalomedullary nail group and 12/15 patients in the PFN group. This was also comparable and not statistically significant. Weiguang $\mathrm{Yu}$ et $a l$. in their study comparing InterTAN nail and PFN antirotation for treatment of unstable intertrochanteric fractures, ${ }^{[18]}$ difference between Harris hip scores was found to be statistically insignificant between the groups, like in our study.

We have known from before that absence of good reduction achieved during surgery results in poor prognosis for the hip fracture ${ }^{[1,19]}$

According to Baumgaertner criteria, good reduction was achieved in almost all cases of both the groups in our study. One of the well-known complications post-surgery is screw cut out which require revision surgery. Reucker et al. in their study with the Intertan nail found varus collapse and screw cut out in $5 \%$ cases ${ }^{[16]}$. And in study done by Schipper et al. for the PFN found varus collapse and screw cut out in $7 \%$ cases ${ }^{[20]}$. In other studies, the cut-out rates for PFN have been documented between $0.6-8 \%{ }^{[13,21]}$.

Basically, improper screw placement in the femoral head was found to be the reason for this complication ${ }^{[16,20]}$. In our study we did not find any cases of screw cut out in both the groups as lot of stress was placed on anatomic reduction and correct screw placement in the femoral head. It has been a well-known fact that implant positioning and proper surgical technique play a more pivotal role in eliminating this problem rather than the implant itself.

Basically, the change in the Twin interlocking nail is the addition of the interlocking cephalomedullary screws to prevent $\mathrm{Z}$ and reverse $\mathrm{Z}$ effect. In our study, we noticed $\mathrm{Z}$ effect in 1 case of PFN group and none in the Twin interlocking nail group.

Many studies have reported many instances of secondary femoral shaft fractures (around 17\%) due to the impingement of the short intramedullary device on the anterior cortex. Ruecker et al. encountered no nail impingement or thigh pain problems in patients treated for an intertrochanteric fracture using this nail ${ }^{[16]}$, The diameter of the Twin interlocking cephalomedullary nail tapers from $13.5 \mathrm{~mm}$ in the middle to $11 \mathrm{~mm}$ at the tip, which has a stress dispersion effect on the nail and inner cortex and avoids stress overconcentration around the nail tip. This nail has a split distal tip that reduces overall cross-sectional stiffness of the distal implant. This might give the nail an added advantage. We also didn't encounter this problem in both the groups in our study.

In our study we found that there is no option in the PFN to prevent unchecked sliding which may lead to decreased stability, increasing the chances of varus collapse and lateral impingement. The twin interlocking nail with its unique nested screw design has been shown to achieve controlled compression and the oval profile of the integrated screws locked to the nail and the presence of SET screw prevents further sliding and provides excellent rotational stability ${ }^{[22]}$. This is an advantage if good reduction is achieved and it might prove to be a disadvantage if good reduction and compression is not achieved on table as further sliding at the fracture will not be allowed by the SET screw. Hence takehome message would be to ensure good reduction and compression of fracture intraoperatively before tightening of SET screw.

The main advantage of the twin interlocking nail we found in our study was that controlled linear compression of up to 10 $\mathrm{mm}$ could be achieved intraoperatively to ensure better reduction of the fracture.

This study of ours is not without limitations. Ours was a study with short term outcome. Long term studies and RCTs are required. More Studies with use of this nail in osteoporotic bone needs to be done. Implant removal in the patients with twin interlocking cephalomedullary nail if needed due to multitude reasons can be challenging. And the proximal diameter of the twin interlocking cephalomedullary nail is around $16 \mathrm{~mm}$ and it can be difficult to place in short statured individuals. 


\section{Conclusion}

Unstable intertrochanteric fractures of the femur are very common and there has been a debate regarding the treatment of these fractures. The intramedullary nails which are biomechanically better have been preferred. There is no clear winner in the choice of the implant in the treatment of this fracture.

We would like to conclude that Twin interlocking Cephalomedullary nail is a viable alternative for unstable intertrochanteric fractures of femur to the PFN which has been extensively in the past from an Indian perspective.

\section{References}

1. Davis TR, Sher JL, Horsman A, Simpson M, Porter BB, Checketts RG. Intertrochanteric femoral fractures. Mechanical failure after internal fixation. J Bone Joint Surg Br 1990;72:26-31.

2. Simpson AH, Varty K, Dodd CA. Sliding hip screws: modes of failure. Injury 1989;20:227-31.

3. Bridle SH, Patel AD, Bircher M, Calvert PT. Fixation of intertrochanteric fractures of the femur. A randomised prospective comparison of the gamma nail and the dynamic hip screw. J Bone Joint Surg Br 1991;73:330-4.

4. Yu J, Zhang C, Li L, Kwong JSW, Xue L, Zeng X, Tang $\mathrm{L}$, Li $\mathrm{Y}$, Sunb $\mathrm{X}$. Internal fixation treatments for intertrochanteric fracture: a systematic review and metaanalysis of randomized evidence. Sci Rep 2015.

5. Queally JM, Harris E, Handoll HHG, Parker MJ. Intramedullary nails for extracapsular hip fractures in adults. Cochrane Database Syst Rev 2014.

6. Matre K, Havelin LI, Gjertsen JE, Espehaug B, Fevang JM. Intramedullary nails result in more reoperations than sliding hip screws in two-part intertrochanteric fractures. Clin Orthop Relat Res 2013;471(4):1379-86.

7. Matre K, Havelin LI, Gjertsen JE, Vinje T, Espehaug B, Fevang JM. Sliding hip screw versus IM nail in reverse oblique trochanteric and subtrochanteric fractures. A study of 2716 patients in the Norwegian Hip Fracture Register. Injury 2013;44(6):735-42.

8. Matre K, Vinje T, Havelin LI, Gjertsen JE, Furnes O, Espehaug B, Kjellevold SH, Fevang JM. TRIGEN INTERTAN intramedullary nail versus sliding hip screw: a prospective, randomized multicenter study on pain, function, and complications in 684 patients with an intertrochanteric or subtrochanteric fracture and one year of follow-up. J Bone Joint Surg Am 2013;95(3):200-8.

9. Gavaskar AS, Tummala NC, Srinivasan P, Gopalan H, Karthik BSS. Helical Blade or the Integrated Lag Screws. Journal of Orthopaedic Trauma 2018;32(6):274-277.

10. Baumgaertner MR, Curtin SL, Lindskog DM, Keggi JM. The value of the tip-apex distance in predicting failure of fixation of peritrochanteric fractures of the hip. J Bone Joint Surg Am 1995;77:1058-64.

11. Radford PJ, Needoff M, Webb JK. A prospective randomised comparison of the dynamic hip screw and the gamma locking nail. J Bone Joint Surg Br 1993;75:78993.

12. Boldin C, Seibert FJ, Fankhauser F, Peicha G, Grechenig W, Szyszkowitz R. The proximal femoral nail (PFN)-a minimal invasive treatment of unstable proximal femoral fractures: a prospective study of 55 patients with a follow-up of 15 months. Acta Orthopaedica Scandinavica 2003;74(1):53-58.

13. Al-Yassari G, Langstaff RJ, Jones JW, Al-Lami M. The AO/ASIF proximal femoral nail (PFN) for the treatment of unstable trochanteric femoral fracture. Injury 2002;33(5):395-99.

14. Gadegone WM, Salphale YS. Proximal femoral nail-an analysis of 100 cases of proximal femoral fractures with an average follow up of 1 year. International orthopaedics 2007;31(3):403-08.

15. Brumback RJ, Uwagie-Ero S, Lakatos RP, Poka A, Bathon GH, Burgess AR. Intramedullary nailing of femoral shaft fractures. Part II: Fracture-healing with static interlocking fixation. The Journal of Bone and Joint Surgery (Am) 1988;70(10):1453-62.

16. Ruecker AH, Rupprecht $M$, Gruber M, Gebauer M, Barvencik F, Briem D, Rueger JM. The treatment of intertrochanteric fractures: results using an intramedullary nail with integrated cephalocervical screws and linear compression. J Orthop Trauma 2009;23:22-30.

17. Min WK, Kim SY, Kim TK, Lee KB, Cho MR, Ha YC, Koo KH. Proximal femoral nail for the treatment of reverse obliquity intertrochanteric fractures compared with gamma nail. Journal of Trauma and Acute Care Surgery 2007;63(5):1054-60.

18. Yu W, Zhang X, Zhu X, Hu J, Liu Y. A retrospective analysis of the InterTan nail and proximal femoral nail anti-rotation-Asia in the treatment of unstable intertrochanteric femur fractures in the elderly. Journal of orthopaedic surgery and research 2016;11(1):10.

19. Parker MJ. Cutting-out of the dynamic hip screw related to its position. J Bone Joint Surg Br 1992;74:625.

20. Schipper IB, Steyerberg EW, Castelein RM, Van der Heijden FH, den Hoed PT, Kerver AJ, Van Vugt AB. Treatment of unstable trochanteric fractures. Randomised comparison of the gamma nail and the proximal femoral nail. J Bone Joint Surg Br 2004;86:86-94.

21. Ballal MS, Emms N, Ramakrishnan M, Thomas G. Proximal femoral nail failures in extracapsular fractures of the hip. Journal of Orthopaedic Surgery 2008;16(2):146-49.

22. Santoni B, Nayak A, Cooper S et al. Comparison of Femoral Head Rotation and Varus Collapse Between a Single Lag Screw and Integrated Dual Screw Intertrochanteric Hip Fracture Fixation Device Using a Cadaveric Hemi-Pelvis Biomechanical Model. J Orthop Trauma 2016;30:164-169. 